\title{
Comparison of two analytical methods validated for the determination of volatile compounds in virgin olive oil: GC-FID vs GC-MS
}

\author{
R. Aparicio-Ruiz , D.L. García-González ${ }^{\mathrm{b}}$, M.T. Morales $^{\mathrm{a}}$, A. Lobo ${ }^{\mathrm{b}}$, I. Romero ${ }^{\mathrm{b}}$ \\ a Department of Analytical Chemistry. Universidad de Sevilla. c/ Prof. García González 2, - 41012- \\ Sevilla, Spain. \\ ${ }^{b}$ Instituto de la Grasa (CSIC), Campus Universidad Pablo de Olavide - Edificio 46, Ctra. de Utrera, km. 1 \\ -41013-Sevilla, Spain
}

*Author to whom correspondence should be sent.

E-mail: iromero@ig.csic.es; Tel: +34 954611550 


\section{ABSTRACT}

The utility of volatile compounds to explain virgin olive oil aroma descriptors is fully accepted and demanded by the olive oil sector. However, the methodology, and particularly the kind of detector to be used, is a matter of discussion because the high number of volatiles and their different nature. The SPME-GC-MS method has recently been validated for the most relevant volatiles but SPME-GC-FID method still needs to be validated to evaluate its performance in this application. A comparison between these two GC methods in determining 26 volatiles has been carried out in terms of analytical quality parameters (repeatability, intermediate precision, calibration curves, limits of detection and quantification, linear working ranges, selectivity and sensitivity). Good selectivity, linearity and higher upper values of the working range are the main advantages of SPME-GC-FID versus low bottom values of working ranges, better sensitivity and lower limits of detection and quantification of SPME-GC-MS. The limit of blank associated to each individual volatile was also determined and it allowed perfecting the empirical limit of detection. This procedure was carried out for SPME-GC-FID, which resulted in 21 volatiles with empirical limits of detections lower than their odor thresholds, and hence they can be used as markers of virgin olive oil sensory descriptors. Finally, with all the analytical quality parameters checked, a practical example of the ability of the volatiles quantified by SPME-GC-FID to discriminate the different categories (extra-virgin, virgin and lampante) and their main aroma descriptors is also provided.

Keywords: virgin olive oil, volatiles, SPME, gas chromatography, analytical quality parameters, odor threshold. 


\section{INTRODUCTION}

The sensory assessment of virgin olive oil categories is currently called into doubt by olive oil market actors despite the international regulations are very strict on how implement the official method so called Panel Test [1,2] and some improvements are being explored [2,3].

There is a strong demand for alternative or complementary methods to the sensory assessment that must be based on chemical compounds responsible for odor sensory descriptors described into the regulations. Those compounds cannot be but volatile compounds because there is a causal relationship between their concentrations and the intensity of the sensory descriptors perceived by assessors or consumers.

Interests - like money and prestige of producers - are at stake because virgin olive oil (VOO) categories have very different commercial values. Thus, analytical methods based on the quantification of volatiles should be subjected to a performance testing to determine their analytical quality parameters prior to being proposed as a standard method to support quality assessment. For this purpose, however, not all of the volatiles have to be quantified but only those responsible for sensory defects as the main problem concerns the qualification of an olive oil as virgin or extra-virgin [4] after the identification of these off-flavors. Thus, an EVOO (Extra Virgin Olive Oil) has to be qualified with sensory descriptors appreciated by consumers and sensory assessors (e.g., fruity, green) and no defective sensory perceptions can be perceived when smelled and tasted (i.e. rancid, vinegary). Sensory qualifiers for VOO are, however, those of EVOO although a very low intensity of any undesirable sensory descriptor is accepted.

The main problem when analyzing volatile compounds is the complexity of VOO aroma due to the large number of volatiles, the great differences in their concentrations (from $\mu \mathrm{g} / \mathrm{kg}$ to $\mathrm{mg} / \mathrm{kg}$ ) [5] and the different functional groups of the volatiles to be quantified. 
Different analytical techniques have been proposed for the quantification of VOO volatile compounds, most of them being based on their pre-concentration prior to the analysis. The most implemented technique in analytical laboratories is headspace-solid-phase micro-extraction (HSSPME) and gas chromatography with flame ionization detector (FID) or mass spectrometry (MS) detector [6-9]. Aside the reasons for the success of SPME in the quantification of VOO volatiles [5], which is not exempt of some disadvantages [7], the current discussion is focused on the decision about the $\mathrm{GC}$ detector for quantifying VOO volatile compounds.

The selection of the detector (GC-FID vs. GC-MS) is crucial but it should not be only influenced by the instrumental cost or the readiness for volatile identification. Thus, the decision making should be based on the values of their analytical quality parameters when quantifying the volatiles responsible for VOO sensory descriptors (mostly undesirable odors) since the objective of the analytical measurements is to achieve reliable and accurate data, and avoid speculation about the results.

There is not any publication reporting a systematic comparison of validation results for the application of analysis of VOO volatiles using FID and MS detectors. Thus, the aim of this study is the validation of SPME-GC-FID for the quantification of VOO volatiles as well as the comparison of its results with those already obtained with the same samples by means of SPMEGC-MS. The results of both validations and their comparison are useful to know the advantages and disadvantages of the detectors in a continuous control of VOO sensory defects, which is still a challenge in VOO quality and authentication [10]. This comparison in validation results has to be carried out compound by compound since the values of the analytical parameters are greatly linked to the particular properties of each compound, such as functional group, volatility, affinity to the fiber and response in the detector. Thus, there is not an absolute answer to select the best detector and the decision making in selecting the best detector, either FID or MS, will depend on 
the particular volatile compounds that are considered as analytical targets and their associated analytical parameters. In this work, we have selected volatile compounds that have been identified as quality markers in previous studies [5]. Additionally, the study was particularly focused on the SPME-GC-FID, and a procedure was suggested to further evaluate the actual limits of detection and to compare them with the odor threshold for assuring a correct chemicalsensory interpretation.

\section{MATERIALS AND METHODS}

\subsection{Samples}

A lampante virgin olive oil (LVOO) sample, provided by the International Olive Oil Council (IOC), characterized with rancid defect (median of defects $\sim 5$ ), and extra-virgin olive oil (EVOO) var. Hojiblanca from Sierra de Yeguas (Málaga) were used for the precision and selectivity study. An odorless refined olive oil (Aceites del Sur, S.L.) was used for the determination of accuracy, linearity and limits of detection and quantification to which different concentrations of volatile standards were added $(0.05,0.10,0.20,0.30,0.50,1.00,3.00,6.00$, $10.00,15.00 \mathrm{mg} / \mathrm{kg}$ ) from a stock solution of $20 \mathrm{mg} / \mathrm{kg}$. All those samples were analyzed by HSSPME-GC-FID (henceforth, GC-FID) and, previously, by HS-SPME-GC-MS (henceforth, GCMS) [8]; the resulting values of the statistical parameters for the validation of analytical methods were used in the comparative study.

Another set of samples, with lower concentration, from 0.01 to $1.00 \mathrm{mg} / \mathrm{kg}$ was analyzed by GC-FID for a further evaluation of the limit of detection of each volatile. In this evaluation, the standard deviation (from 7 replicates) of the signal associated to the lowest concentration of each volatile was determined in order to check whether the lowest concentration of each individual volatile can be easily distinguished from blank chromatograms. The full range (0.01- 
$1.00 \mathrm{mg} / \mathrm{kg}$ ) was split into four sub-ranges - 0.01-0.05, 0.02-0.10, 0.10-0.50, and $0.10-1.00 \mathrm{mg} / \mathrm{kg}$ -. For a given volatile, one of these four ranges was selected as the most appropriate depending on the previously determined limit of blank [11,12]. A set of 20 chromatograms of blank analyses carried out for 6 months was used for calculating the limit of blank individually associated to each one of the volatiles determined for this work.

A set of 22 virgin olive oil (VOO) samples were analyzed by GC-FID once the method was validated. Samples were characterized with the main sensory defects by OleoEstepa SCA (Estepa, Spain) sensory panel following the standard method for the organoleptic assessment of VOO described by the International Olive Council [1].

\subsection{Reagents}

Ethyl acetate (99.5\%), butanoic acid (99\%), propanoic acid (99.5\%), pentanoic acid (99\%), 1-butanol (99\%), 2-butanol (99.5\%), 2-heptanone (98\%), (E)-2-heptenal (98\%), hexanal (98\%), 1-hexanol (98\%), (E)-2-hexenal (97\%), 3-methyl-butan-1-ol (99.5\%), nonanal (95\%), octanal (98\%), pentanal (98\%), octane (99\%) and 1-penten-3-one (98\%) were purchased from Fluka (Zwijndrecht, The Netherlands). Acetic acid (99\%), 2-heptanol (98\%), 1-octen-3-ol (98\%) and ethanol (96\%) were purchased from Sigma-Aldrich (St. Louis, MO). Heptanal (95\%), (E)-3hexen-1-ol (98\%), 6-methyl-5-hepten-2-one (99\%), 3-octanone (97\%) and 3-pentanone (99.5\%) were purchased from Panreac (Barcelona, Spain).

\subsection{Determination of volatile compounds by HS-SPME-GC-FID and HS-SPME-GC-MS}

The sample preparation was carried out according to the method applied in previous works [8]. The concentration step was carried out on a Combipal (CTC Analytics AG, Zwingen, Switzerland). An olive oil sample (2 g) was placed in a $20 \mathrm{~mL}$ glass vial, tightly capped with polytetrafluoroethylene (PTFE) septum, and left for $10 \mathrm{~min}$ at $40{ }^{\circ} \mathrm{C}$ to allow for the equilibration 
of the volatiles in the headspace. After the equilibration time, the septum covering each vial was pierced with an SPME needle, and the fiber was exposed to the headspace for $40 \mathrm{~min}$. The SPME fiber (1 cm length and 50/30 $\mu \mathrm{m}$ film thickness) was purchased from Supelco (Bellefonte, PA), and it was endowed with the Stable Flex stationary phase of divinylbenzene/carboxen/polydimethylsiloxane (DVB/CAR/PDMS). The fiber was previously conditioned following the instructions of the supplier.

The volatiles adsorbed by the fiber were thermally desorbed in the hot injection port of a Varian 3900 gas chromatograph (Palo Alto, CA) in purge valve off mode (splitless mode) for 5 min at $260{ }^{\circ} \mathrm{C}$. The gas chromatograph was equipped with a TR-WAX capillary column $(60 \mathrm{~m} \times$ $0.25 \mathrm{~mm}$ i.d., $0.25 \mu \mathrm{m}$; Teknokroma, Spain) and a flame ionization detector (FID). The carrier gas was hydrogen at a flow rate of $1.5 \mathrm{~mL} / \mathrm{min}$. The oven temperature was held at $40{ }^{\circ} \mathrm{C}$ for 10 min and then programmed to rise at $3{ }^{\circ} \mathrm{C} / \mathrm{min}$ to a final temperature of $200{ }^{\circ} \mathrm{C}$. The signal was recorded and processed with the WorkStation (v6.41) software. Each sample was analyzed in duplicate.

Identification of the volatile compounds was first carried out by mass spectrometry and later checked with standards as described in previous works $[8,13]$. Quantification was done with internal standard (4-methyl-2-pentanol).

The same samples were analyzed with GC-MS (7820A Agilent Technologies gas chromatography coupled to a Series MSD 5975 Agilent Technologies mass spectrometry) in a previous work [8] by applying the same sample preparation procedure, the kinds of fiber and column, and the chromatographic conditions used in the GC-FID method described above. Then, the only difference of the method was the detector used (FID vs. MS) and the flow rate of carrier gas $(1.5 \mathrm{~mL} / \mathrm{min}$ vs. $1 \mathrm{~mL} / \mathrm{min})$. 


\subsection{Statistical Analysis}

Multivariate algorithm of Principal Component Analysis (PCA) was applied by means of Statistica 8.0 (Statsoft, Tulsa, OK). PCA was used because it is an unsupervised statistical procedure that does not foreseen results with the set of independent variables. The selection of independent variables was carried out with chemical parameters (odor threshold and limit of detection) but the relationship between volatiles and olive oil categories (LVOO, VOO and EVOO) was checked with Brown-Forsythe test as it provides information about significant differences $(\mathrm{p}<0.05)$ distinguishing olive oil categories even when the raw scores deviate significantly from the normal distribution.

\section{RESULTS AND DISCUSSION}

\subsection{Method validation}

The validation of analytical methods is the only procedure to determine the usefulness of the methods for a particular application. Thus, method validation is the process of establishing the performance characteristics and limitations of a method to check that it is adequate for solving a particular analytical problem. In the analytical problem of quantifying volatiles in VOO, the most common analytical approach is based on gas chromatography. However, there is not a unanimous agreement about the detector. Although a majority of laboratories uses FID as detector, there are many laboratories, mainly in research and governmental institutions, which detector is MS. Economical reasons and availability are always weighed when selecting a detector, and for that reason a FID detector may prevail over MS. However, once the analytical problem is defined, the analytical quality of the measurements must be among the main criteria for selecting a definitive 
method. Thus, internal validation of both analytical methods (GC-FID and GC-MS) was carried out by the determination of the analytical quality parameters: precision, linearity, limits of detection and quantification, working range, accuracy, selectivity and sensitivity.

\subsubsection{Precision}

Two types of precision - repeatability and intermediate precision [14] - were determined for GC-FID and the results were compared with a previous study of the authors with GC-MS [8]. Repeatability of each one of 26 volatiles characterizing the two samples (EVOO and LVOO) was determined in working sessions of 8 replicates (intra-day analyses) while intermediate precision (inter-day analyses) of each volatile was determined by the analysis of the samples in 8 nonconsecutive working sessions (one per month). The two samples used for this study were from the two extreme VOO categories, from the highest (EVOO) to the lowest (LVOO) quality. It means that volatiles contributing to undesirable sensory perceptions will be at high concentrations in LVOO and at very low or not detected in EVOO. On the contrary, volatiles contributing to positive sensory perceptions will be at high concentration in EVOO. Thus, by analyzing these two samples, two different concentrations were considered for each volatile compound in the precision study, which is more informative than a single concentration value coming from one selected sample. The results were expressed as relative standard deviation (RSD\%) in Table 1. This table shows that the values of repeatability for GC-FID are good enough $(<10 \%)$ for 22 (out of 26) volatiles, and only one compound (2-butanol) showed a RSD\% value slightly higher than $15 \%$. In terms of kind of samples, the repeatability is similar when analyzing LVOO and EVOO, the mean values being $7.26 \%$ vs. $6.60 \%$, respectively. However, the number of volatiles with $\mathrm{RSD} \%$ lower than $10 \%$ is higher in EVOO than in LVOO (22 vs. 18). The volatiles that showed 
RSD\% higher than $10 \%$ were not associated to a particular functional group or to the fact that they were responsible for negative attributes, which means higher concentrations in LVOO.

The results from the intermediate precision for GC-FID were poorer (higher RSD\%) than the within-day repeatability, as expected (Table 1). However, the values of intermediate precision were similar for EVOO and LVOO samples in terms of RSD\% mean values (8.76\% vs. 8.92\%) and number volatiles with values lower than $10 \%$ (17 vs. 15). The results of both repeatability and intermediate precision seems to show that the quality category of the sample does not affect the results when using CG-FID despite a LVOO is expected to have more volatiles and at higher concentration than an EVOO [15]. However, the results obtained with CG-MS analyzing the same samples are different since the volatile composition associated to the quality category seems to have an effect on precision. In the case of the repeatability of GC-MS, a better precision (lower RSD\%) was also lower working with LVOO (4.25\%) than with EVOO (9.85\%); a similar ratio was observed when analyzing its intermediate precision $(14.86 \%$ for EVOO vs. $5.95 \%$ for LVOO). As the concentration of volatiles in both methods is carried out with SPME applying the same procedure, the effect of competition among volatiles during that step is not a reason for these differences in the results [7]. Others reasons like selectivity and sensitivity of FID and MS detector [16], as described below, are possible reasons for these differences in precision together with a higher variation in the ionization source when analyzing volatiles, which concentrations widely vary from $\mu \mathrm{g} / \mathrm{kg}$ to $\mathrm{mg} / \mathrm{kg}$.

\subsection{2.- Linearity}

The ability of an analytical method to give responses that are proportional to the amounts of an analyte in a sample determines its linearity [17]. In quantitative analytical methods, a linearity study is previous to determine the concentration range or the property values of the 
analyte over which the method is intended to be applied. Table 2 shows the linearity parameters studied for the GC-FID and GC-MS methods. Considering a wide concentration range of 0.1-15.0 $\mathrm{mg} / \mathrm{kg}$, the squared adjusted regression coefficient $\left(\mathrm{R}^{2}\right.$ adj $)$ for GC-FID $(\geq 0.99)$ were slightly better than GC-MS (from 0.96 to 0.99). These coefficients, however, do not always guarantee the linearity of the calibration curve [18], and information of the relative standard deviation of the regression $\left(\mathrm{RSD}_{\mathrm{b}}\right)$ and the distribution of regression residuals are needed to reach a plausible conclusion [17].

Table 2 shows the $\mathrm{RSD}_{\mathrm{b}}$ for the compounds quantified by GC-MS and GC-FID. If a RSD value lower than $5 \%$ is considered to be necessary to confirm tentatively the linearity, only two volatiles quantified by GC-FID, 3-pentanone and E-3-hexen-1-ol, did not meet this requirement, which means their linearity would not be guaranteed in the analyzed range $(0.1-15.0 \mathrm{mg} / \mathrm{kg})$. E-3hexen-1-ol also showed a RSD bigher than 5\% when working with GC-MS (Table 2) together with 5 more volatiles: ethyl acetate, nonanal and 3 acids (acetic, butanoic and pentanoic).

The analysis of residuals was carried out considering that a calibration conforms to linearity if also it complies with the following properties: residual values should show no trend, residuals should be uniformly distributed (random distribution) and the numbers of negative and positive residual values have to be approximately equal. The analysis of residuals was stricter in terms of linearity since only 11 volatiles $(42 \%)$ accomplished with the requested properties in the whole concentration range (0.1-15.0 $\mathrm{mg} / \mathrm{kg}$ ) for GC-FID (Table 2). In the case of GC-MS, only two compounds (1-butanol and 6-methyl-5-hepten-2-one) showed a residual plot that met the required properties described above for the whole concentration range of $0.1-15.0 \mathrm{mg} / \mathrm{kg}$ (Table 2).

The importance of residuals is underlined in Figure 1A where 6-methyl-5-hepten-2-one $\left(\mathrm{R}^{2}{ }_{\text {adj }}=0.999\right.$ and $\left.\mathrm{RSD} \%=1.18\right)$ shows a linear behavior in the full range $(0.1-15.0 \mathrm{mg} / \mathrm{kg}) \mathrm{while}$ 
ethyl acetate $\left(\mathrm{R}^{2}{ }_{\text {adj }}=0.999\right.$ and $\left.\mathrm{RSD} \%=1.56\right)$ do show a residual plot with a clear trend that points out a lack of linearity (Figure 1B). In this latter case, linearity would be acceptable if two separate concentration ranges are considered (e.g., 0.1-1.0 mg/kg and 3.0-15 mg/kg). Thus, comparing RSD values and the residual plots of GC-FID and GC-MS, these results point out the high linear range of the FID detector, which is among the largest of all GC detectors [16].

Since the pre-concentration step based on SPME was the same in both methods, this difference in linearity between the two methods could be attributed to a higher sensitivity of MS detector for low concentrations compared with FID [19] rather than to a limitation of the capacity of the adsorbent material of the fiber at high concentrations. However, most of the volatile compounds present in the VOO are in concentrations lower than 3.0-6.0 mg/kg [20], these values being within the linear range of work. Furthermore, there can be more than one lineal segment inside the whole range $(0.05$ to $15.00 \mathrm{mg} / \mathrm{kg})$ or the response in the whole range be perfectly adjusted to a non-lineal equation. Thus, the non-linear behavior of the volatile data in the whole proposed range of $0.1-15.0 \mathrm{mg} / \mathrm{kg}$ can be fitted to a logarithmic model equation $(\mathrm{Y}=\mathrm{a}+\mathrm{b} \times \log \mathrm{X})$. Ethyl acetate, 3-pentanone, 1-butanol and E-3-hexen-1-ol quantified by GC-MS and E-3-hexen1-ol and 3-pentanone quantified by GC-FID improved their regression coefficients when applying the logarithmic equation.

\subsection{3.- Limits of detection and limits of quantification}

The lowest concentration of the analyte that can be detected by the method at a specified level of confidence is named limit of detection (LOD) [21]. LOD refers to the qualitative aspect of the method but does not refer to whether the analyte can be reliably quantified. For this purpose, the limit of quantification (LOQ) is additionally required. LOQ is defined as the minimum amount of analyte that can be quantified with an acceptable level of precision and 
accuracy [21]. The values of these statistical parameters can be determined from the relationship between the standard deviation of the regression and the slope of the calibration curve [22] or simply by the empirical information. The second option [23] is based on the assumption that the area of the compounds should be at least three times higher than the signal-to-noise ratio (LOD). In this study, LOD and LOQ were calculated using the first option considering the ratio between the standard deviation of the regression and the slope of the calibration multiplied by three for LOD and by ten for LOQ.

Table 2 shows the data obtained in the calculation of LOD and LOQ for each volatile analyzed by GC-FID. A percentage of $65 \%$ of the volatiles showed LOD lower than $1.0 \mathrm{mg} / \mathrm{kg}$ ( $81 \%$ when working with GC-MS). Analyzing the results in terms of the main series of volatiles (aldehydes, alcohols, ketones, acids and esters), the lowest and highest values are very similar for all the series. Only 2-butanol showed an exceptional value since its LOD was very low (0.1 and $0.05 \mathrm{mg} / \mathrm{kg}$ for GC-MS and GC-FID, respectively) compared with the rest of compounds. Comparison of results for GC-MS and GC-FID points out that the former technique has, in general, LOD values lower than GC-FID although some compounds show similar results for both techniques (e.g. 2-heptanol, 6-methyl-5-hepten-2-one, E-3-hexen-1-ol, acetic acid, pentanoic acid) or they were of the same order. Thus, for example, nonanal shows the highest LOD among the aldehydes for both techniques $(1.14 \mathrm{mg} / \mathrm{kg}$ and $2.41 \mathrm{mg} / \mathrm{kg}$ for GC-MS and GC-FID, respectively), and the acids have also LOD values of the same order with the exception of propanoic acid (Table 2).

Although the range of these parameters is wide enough, the analyst should keep in mind the ample variability in the concentration of the volatiles (from $\mu \mathrm{g} / \mathrm{kg}$ to $\mathrm{mg} / \mathrm{kg}$ ) [5] and the number of these compounds analyzed. In general terms, the results agree with the fact that GC- 
MS generally provide lower LOD and LOQ values than GC-FID because it is able to perform quantitative analyses even below the level of $\mu \mathrm{g} / \mathrm{kg}$ [16].

\subsection{4.- Working range.}

Once the linearity and the LOD/LOQ were studied for each volatile, the working range of them was determined. Table 2 shows the working ranges for the volatiles, which was defined as the range between the minimum value of LOQ and the highest concentration tested with good linearity [18]. In the case of GC-FID, $42 \%$ of the working ranges reached the maximum value of the studied concentrations $(15 \mathrm{mg} / \mathrm{kg})$ while $31 \%$ reached a medium value $(6 \mathrm{mg} / \mathrm{kg})$. By contrast, $62 \%$ of the working ranges for GC-MS had a maximum value of $3 \mathrm{mg} / \mathrm{kg}$. On the other hand, the ratio between the maximum and minimum values of the working ranges (Table 2) provided a useful information to understand the width of these ranges and the ability of the method for quantifying volatiles compounds at different concentrations. Thus, the mean values of these ratios were very similar for both technique, 5.9 for GC-MS and 5.3 for GC-FID. The minimum values observed was also similar, 1.1 vs. 1.5 for GC-MS and GC-FID respectively. Heptanal showed a particularly high ratio for both methods, overall in the case of GC-MS (57.69). If heptanal is omitted, no differences associated to particular functional groups were observed between GC-FID and GC-MS, except for the acids, which working range is much better for GC-FID.

\subsection{5.- Apparent accuracy}

Apparent accuracy expresses the ratio between the area determined by GC-FID and GCMS and transformed into concentration $(\mathrm{mg} / \mathrm{kg})$ with the calibration curve of each volatile and the standard value [24]. This way of evaluating accuracy has been successfully applied in GCFID and its formula [8] allows calculating the experimental value of t-test $\left(\mathrm{t}_{\exp }\right)$ to be compared 
with the tabulated value $\left(\mathrm{t}_{\mathrm{tab}}\right)$ from a two-sided $\mathrm{t}$-table for $\alpha=0.02$ and $\mathrm{n}$ - 1 degrees of freedom. None of the twenty-six quantified volatiles by GC-FID did show poor accuracy; in fact, in all the cases $t_{\exp } \leq t_{\text {tab }}$.

\subsection{6.-Selectivity}

The selectivity parameter determines whether a chromatographic peak is only due to the analyte of interest and not to the presence of interferences in the sample. The chromatographic profile of VOO volatiles with numerous compounds is a paradigm for studying selectivity as not always there is enough baseline between peaks. The selectivity was calculated by means of the formula of chromatographic resolution in which the retention times and the width of two adjacent peaks are considered [18,25]. A chromatographic resolution higher than 1.0 means that there is not overlap between two adjacent peaks and the signal produced during the measurement of the peak under study is only due to the analyte $[18,25]$. A chromatographic resolution higher than 1.5 means the peaks are completely resolved and additionally there is a baseline between them [22]. Table 2 shows that only two peaks quantified by GC-FID showed a chromatographic resolution lower than 1.0 and they were not fully resolved with respect to its previous and next peaks (pentanal and 2-heptanone) while other five peaks are not well resolved with either their previous or next peaks (3-pentanone, hexanal, heptanal, E-3-hexen-1-ol, nonanal).

All the peaks are well resolved when using GC-MS (resolution $\geq 1.0$ ), which means that seemingly a GC-MS method have higher selectivity than a GC-FID method. However, there are more undetected compounds with GC-MS than GC-FID (6 vs. 1), and the mean chromatographic resolutions (for previous and next peaks) of GC-MS (4.5 and 3.9 for previous and next peaks respectively) are also lower than GC-FID (5.8 and 6.7 for previous and next peak respectively). 


\subsection{7.-Sensitivity}

The sensitivity is the slope value of the calibration curve of chromatographic areas against the concentration of the volatiles. Table 2 shows the sensitivity values for GC-MS and GC-FID. Considering the chemical series for GC-FID, the average value of sensitivity in acids was the lowest $\left(0.34 \times 10^{4}\right)$, followed by aldehydes $\left(0.63 \times 10^{4}\right)$. However, the sensitivity of hexanal was much higher $\left(1.16 \times 10^{4}\right)$. The average value of sensitivity of ketones and alcohols were higher $\left(1.29 \times 10^{4}\right.$ and $1.18 \times 10^{4}$, respectively). In terms of individual compounds, the lowest values corresponded to 2-heptanol $\left(0.07 \times 10^{4}\right)$, octanal $\left(0.07 \times 10^{4}\right)$, 6-methyl-5-hepten-2-one $\left(0.04 \times 10^{4}\right)$ and acetic acid $\left(0.08 \times 10^{4}\right)$. Three of the highest sensitivity, in GC-FID, corresponded to ethyl acetate $\left(2.45 \times 10^{4}\right), 3$-pentanone $\left(3.55 \times 10^{4}\right)$ and 1-butanol $\left(2.65 \times 10^{4}\right)$. In the case of GC-MS, the first two volatiles were also those with highest sensitivity $\left(2.72 \times 10^{7}, 3.35 \times 10^{7}\right)$ together with octane $\left(2.80 \times 10^{7}\right)$. In general terms, GC-MS showed higher sensitivity than GC-FID $\left(\times 10^{7}\right.$ vs. $\times 10^{4}$ ), which matches with its good performance when working at low concentrations [19].

\section{2.- Explaining VOO sensory perceptions with volatiles from Chemistry: cross-matching of limits of detection and odor thresholds.}

The GC-FID validated method has an immediate application in determining the volatiles responsible for aroma descriptors as perceived by consumers and panelists. The concentrations of volatiles, in fact, determine whether they contribute to the aroma and they are perceived by consumers always if they overpass their odor threshold. The latter is the minimum concentration in a matrix of refined olive oil at which the aroma is perceived by trained assessors (panelists). Thus, the level of contribution of any volatile to aroma - so-called odor activity value (OAV) results from the ratio between its concentration in VOO and its odor threshold (OT). The OT values of the volatiles contributing to $\mathrm{VOO}$ aroma commonly vary from $\mu \mathrm{g} / \mathrm{kg}$ to $\mathrm{mg} / \mathrm{kg}$. It means 
that analytical techniques can compete with trained assessors if, and only if, LODs are lower than, or similar to, OTs of each individual volatile contributing to VOO aroma. Thus, LOD is one of the most critical parameters when applying volatiles for the early detection of sensory descriptors of VOO sensory assessment from Chemistry.

The minimum concentration of the experiment to build the calibration curves for the previously described analytical parameters was $0.1 \mathrm{mg} / \mathrm{kg}$. This concentration should be higher than the true value of LOD. However, some volatiles (e.g., 2-butanol may have a lower LOD. Thus, an accurate determination of LOD would mean lowering the concentration values in the study to determine the minimum concentration of each volatile that can reliably be distinguished from "analytical noise" or signal produced in absence of volatiles. In other words, the procedure should determine the minimum concentration of the volatiles that can be detected at a known confidence level [26]. Thus, the minimum concentration of each volatile must be determined individually and its resulting chromatographic signal be estimated by GC-FID with a reasonable statistical certainty. The aim is to avoid the case of an apparent identification of a volatile in the chromatogram when it was, in fact, a peak of the noise in the blank signal (false positive). Likewise, having an accurate determination of LOD would also avoid the case of a volatile that is ignored for presenting apparently too low chromatographic areas, while its signal in the chromatogram, is in fact, clearly over the signal to noise ratio and therefore it is due to the presence of the compound (false negative).

The method for estimating a signal from the minimum detectable concentration of each volatile requires previously determining the limit of blank (LOB), which has been formulated as $\mathrm{LOB}=$ mean $_{\text {blank }}+1.645 \times \mathrm{SD}_{\text {blank }}[11,26]$; SD being the standard deviation of the response for several blank runs. This study, on the other hand, has not been focused on only an analyte (volatile), but on numerous ones which are identified along $60 \mathrm{~min}$ of the chromatogram. LOB 
should be calculated individually for all the volatiles, along their corresponding retention times, and its information for determining "mean" and "standard deviation" (SD) determined considering the influence of their nearby peaks. LOB associated to each volatile is displayed in Table 3, and it was calculated from 20 chromatograms of blank samples analyzed for 6 months to consider all possible variations. The normal distribution curve for the blank observations in the vicinity of the retention time of each volatile was check for normality [12]. Thus, LOB included fluctuations, disturbances and instabilities in the flow of carrier to the detector. Results, in terms of chromatographic areas, vary from 1000 to 17822 units; $73 \%$ being lower than 5000 units. The value of each LOB is not but a red line for determining the LOD of each volatile. In fact, LOD of each volatile can be determined according to $\mathrm{LOD}=\mathrm{LOB}+1.645 \times \mathrm{SD}_{\text {low }}$ concentration sample $)[11,27]$, which is one of the possible algorithms for determining this statistical parameter for analytical methods [27]. This algorithm is suggested when determination involves background noise [12] as it occurs in this work.

Prior to the determination of this LOD, the standard deviation (SD) associated to the lowest concentration of each volatile is previously needed. The objective is to get an evidence that a signal associated to the presence of a given compound can be easily distinguished from its associated LOB. Determining this SD involves carrying out the chromatographic determinations of each volatile solved in fully refined olive oil at different concentrations in the vicinity of its LOB. Thus, considering this LOB, and a series of low concentrations, always lower than 1.0 $\mathrm{mg} / \mathrm{kg}$, were tested in GC-FID. These concentration ranges, shown in Table 3, were defined for each volatile compound including those concentrations that produced a chromatographic area near the blank noise. Thus, four ranges were studied: $0.01-0.05 \mathrm{mg} / \mathrm{kg}$ (16 volatiles), 0.02-0.10 $\mathrm{mg} / \mathrm{kg}$ (6 volatiles), $0.05-0.50 \mathrm{mg} / \mathrm{kg}$ (2 volatiles) and $0.10-1.00 \mathrm{mg} / \mathrm{kg}$ (2 volatiles). The experiment in each concentration was repeated 7 times to determine the SD associated with the 
minimum concentration of each volatile that can be easily distinguished from its LOB. It was accepted that SD expressed in area should be at least 1.5 times higher than the mean area of the blank. The determination of SD allowed determining the LOD for each volatile applying the aforementioned equation based on LOB and SD.

Table 3 shows LOD values for each volatile compound. The LODs for identified volatiles are, in general, lower than their corresponding OTs. It means that GC-FID might be an early control tool for detecting sensory descriptors contributing to VOO aroma since the detectable concentrations are lower than the concentrations that can be perceived by a panelist. However, some exceptions were found. Thus, E-2-heptenal, 1-penten-3-one and 1-octen-3-ol showed values of OTs much lower $(\mu \mathrm{g} / \mathrm{kg})$ than their corresponding LOD $(\mathrm{mg} / \mathrm{kg})$. Thus, these three compounds could not be firstly detected by GC-FID compared with trained assessors. On the other hand, 2heptanol, nonanal and butanoic acid showed OTs around 3-times lower than their LODs..

Analyzing these results from a sensory viewpoint, some of the quantified compounds could be identified as markers of sensory defects since their detection is guaranteed with low values of LOD. Previous works have identified some volatile compounds that can be used as indicative of sensory defects in VOO [5]. Thus, nonanal is possibly the best marker of an early warning of oxidation process in VOO [28]. Acids (e.g. propanoic, butanoic and pentanoic) and aldehydes (e.g. hexanal, heptanal and octanal) also increase their concentration during oxidation [13], their LODs being lower than their OTs. Some C8 compounds (i.e. 1-octen-3-ol) are responsible for mustiness/humidity sensory perception while acetic acid is a clear responsible for vinegary perception [13]. Other volatiles are responsible for other undesirable perceptions detected in LVOOs and VOOs [29]. Whichever the volatile that is selected for controlling offflavors in oils, its LOD determined with an accurate procedure should be lower than its OT. 
The LOD values of most of the volatiles proved that these compounds can be detected before they are perceived by any panelist. Then, the next step was centered on checking the usefulness of volatiles for qualifying VOO samples from a sensory viewpoint. VOO aroma is described with different sensory descriptors, which can be clustered in 'desirable' and 'undesirable' by consumers [2,5]. The second set of descriptors is decisive to verify that an oil is EVOO, which means that no undesirable attribute is detected, and no volatile responsible for these attributes is identified. For that reason, proving the absolute absence of these compounds by GC-FID is reliable enough only if their LOD are known and they are compared with their OT. On the other hand, the presence of these compounds at concentrations higher than their OT would mean that this oil belongs to VOO category, which can be qualified with a very slight undesirable sensory perception, or belongs to LVOO category, which oils are qualified with an intense aroma to undesirable sensory descriptors [30].

The ability of distinguishing samples of VOO by their quality was checked with a set of 22 real samples collected from an olive mill, 17 samples being qualified as LVOO as they were characterized with different undesirable descriptors (frostbitten olives, fusty-muddy sediment, hay-wood, musty-humid-earthy, rancid, vinegary-winey). The other 5 samples were EVOO (3) and VOO (2). Table 3 shows the concentrations (mean \pm SD) of 22 olive oil samples. They were clustered in LVOOs, which volatile concentrations were expected to be high, and EVOO+ VOO, with volatiles at lower concentrations if they are not responsible for sensory defects. No volatile was considered to be informative enough for all kinds of defects in VOO [5]. However, multivariate statistical analysis carried out on all the volatiles can inform about the quality of the samples. Thus, the statistical procedure of Principal Component Analysis (PCA) was applied to those volatiles which mean concentrations for any of the groups were higher than their limit of detection (LOD) and their odor thresholds (OT), as displayed in Table 3. Only 11 volatiles 
accomplished with these conditions, five of them contributing to undesirable sensory perceptions (ethyl acetate, 3-methyl-1-butanol, E-2-heptenal, nonanal, acetic acid) and other five to desirable sensory perceptions (2-butanol, hexanal, E-2-hexenal, 1-hexanol, E-3-hexen-1-ol). Analyzing the set of eleven selected volatiles, two of them had OTs lower than their LODs (E-2-heptenal and nonanal) but their concentrations in the two sets were much higher than their LODs (Table 3). Furthermore, volatiles contributing to desirable perceptions - in accordance with their concentration in the samples - have higher concentration in the samples located in the cluster of EVOO+VOO; i.e. 2-butanol, hexanal, E-2-hexenal, 1-hexanol and E-3-hexen-1-ol.

Figure 2 shows the results with a clear differentiation between LVOOs and EVOOs and VOOs. The factor loadings revealed that high concentrations of 3-methyl-1-butanol and ethyl acetate partially explained the differences displayed in Figure 2 between LVOOs qualified with fusty undesirable perceptions and the other LVOOs. The concentration of E-2-heptenal might explain differences between LVOOs qualified with the perception to frostbitten olives and the other LVOOs. The concentration of acetic acid is decisive for explaining the position of wineyvinegary LVOO samples in Figure 2 when compared with LVOOs qualified with sensory perceptions from fermented processes (musty-humid-earthy, fusty-muddy sediment). Finally, the concentration of 1-hexanol is double in EVOOs than VOOs, and the concentration of E-3-hexen1-ol is four times higher in EVOOs than in VOOs. This differences explained that EVOOs and VOOs were plotted separately.

The separation of PCA proved the ability of volatiles assessing on the quality category of the samples. The study of the analytical quality parameters carried out in GC-MS and GC-FID proved that both analytical approaches could be applied since the two methods have advantages and disadvantages. Thus, depending on the volatile being quantified and its concentration, one of the two methods can be selected as the most appropriate. From all the quality parameters, LOD 
was considered as one of the most relevant since it determines whether a quality marker (volatile) responsible for any off-flavor is detected before or after a panelist can perceive it. Thus, the LOD of a volatile, accurately determined, and the comparison with its OT help to know whether its analytical determination by GC might provide information to be interpreted in sensory terms. Thus, the ability of volatiles distinguishing different quality perceptions in VOO categories, confirmed by multivariate statistical analysis of PCA, is supported by analytical quality parameters (Tables 1-3) that avoid unexpected errors. The development of techniques that support panel test is one the challenges of olive oil research [10], and extracting a major knowledge about the candidate techniques and their analytical characteristics, which were studied in this work, can facilitate the development of a standard method.

\section{Acknowledgment}

This work was supported by the Comisión Interministerial de Ciencia y Tecnología (Spanish Government) through the projects AGL2011-30371 and AGL2015-69320-R.

\section{References}

[1] IOC, Sensory analysis of olive oil. Method for the organoleptic assessment of virgin olive oil. COI/T.20/Doc. No 15/Rev. 8/ 2015.

[2] EC, European Commission, Newsletter. Workshop on Sensory Analysis for better quality virgin olive oil. 2 October 2015 Milan, Italy. URL: https://ec.europa.eu/agriculture/sites/agriculture/files/expo-milano-2015/capevents/virgin-olive-oil/proceedings en.pdf.

[3] D.L. García-González, R. Aparicio, Research in olive oil: Challenges for near future. J. Agric. Food Chem. 58 (2010) 12569-12577. 
[4] D.L. García-González, N. Tena, I. Romero, R. Aparicio-Ruiz, M.T. Morales, R. Aparicio, A study of the differences between trade standards inside and outside Europe. Grasas Aceites 68 (2017) e210.

[5] M.T. Morales, R. Aparicio-Ruiz, R. Aparicio, Chromatographic Methodologies: Compounds for Olive Oil Odor Issues, in: R. Aparicio, J. Harwood (Eds.). Handbook of Olive Oil. Analysis and Properties, second ed., Springer, New York, 2013, pp. 261-309.

[6] N. Tena, A. Lazzez, R. Aparicio-Ruiz, D.L. García-González, Volatile compounds characterizing Tunisia Chemlali and Chetoui virgin olive oils. J. Agric Food Chem. 55 (2007) 7852-7858.

[7] C. Oliver-Pozo, R. Aparicio-Ruiz, I. Romero, D.L. García-González, Analysis of Volatile Markers for Virgin Olive Oil Aroma Defects by SPME-GC/FID: Possible Sources of Incorrect Data. J. Agric. Food Chem. 63 (2015) 10477- 10483.

[8] I. Romero, D.L. García-González, R. Aparicio-Ruiz, M.T. Morales, Validation of SPMEGCMS method for the analysis of virgin olive oil volatiles responsible for sensory defect Talanta 134 (2015) 394-401.

[9] L. García-Vico, A. Belaj, A. Sánchez-Ortiz, J.M. Martínez-Rivas, A.G. Pérez, C. Sanz, Volatile Compound Profiling by HS-SPME/GC-MS-FID of a Core Olive Cultivar Collection as a Tool for Aroma Improvement of Virgin Olive Oil. Molecules 22 (2017) 141-153.

[10] EU, Horizon 2020 Work Programme 2014-2015. Olive oil Authentication, 2014. 〈http://ec.europa.eu/research/participants/portal/desktop/en/opportunities/h2020/topics/23 29-sfs-14a-2014.html .

[11] D.A. Armbruster, T. Pry, Limit of blank, limit of detection and limit of quantification. Clin. Biochem. Rev. 29 Suppl 1 (2008) S49-S52.

[12] G.L. Long, J.D. Winefordner, Limit of Detection. A closer look at the IUPAC definition. Anal. Chem. 55 (1983) 712A-724A.

[13] M.T. Morales, G. Luna, R. Aparicio, Comparative study of virgin olive oil sensory defects. Food Chem. 91 (2005) 293-301. 
[14] ISO, Internal Organization for Standardization, 5725. Accuracy (Trueness and Precision) of Measurement Methods and Results. 1994.

[15] D.L. García-González, J. Vivancos, R. Aparicio, Mapping brain activity induced by olfaction of virgin olive oil aroma. J. Agric. Food Chem. 59 (2011) 10200-10210.

[16] D. Rood, Detectors, in: D. Rood (Ed.), The Troubleshooting and Maintenance Guide for Gas Chromatographers. Wiley-Verlag, Weinheim, 2007, pp. 139-185.

[17] R. Aparicio, L.S. Conte, H.-J. Fiebig, Olive Oil Authentication, in: R. Aparicio, J. Harwood (Eds.) Handbook of Olive Oil. Analysis and Properties, second ed., Springer, New York, 2013, pp. 589-653.

[18] R. Boqué, A. Maroto, J. Riu, F.X. Rius, Validation of analytical methods. Grasas Aceites 53(2002) 128-143.

[19] D.O. Sparkman, Z.E. Penton, F.G. Kitson, Gas Chromatography and Mass Spectrometry: A Practical Guide, second ed., Elsevier, Amsterdam. 2011.

[20] D.L. García-González, M.T. Morales, R. Aparicio, Olive and olive oil, in: Y.H. Hui (Ed.), Handbook of Fruit and Vegetable Flavors, Wiley, New Jersey. 2010. pp. 821-847.

[21] EURACHEM, The Fitness for Purpose of Analytical Methods, A Laboratory Guide to Method Validation and Related Topics, B. Magnusson and U. Örnemark (eds.), EURACHEM Secretariat, 2nd ed. 2014.

[22] N.J. Miller, J.C. Miller, Estadística y Quimiometría para Química Analítica, fourth ed. Pearson Education, Madrid. 2002.

[23] J.E. Knoll, Estimation of the limit of detection in Chromatography. J. Chromatogr. Sci. 23 (1985) 422-425.

[24] IUPAC, International Union of Pure and Applied Chemistry, Selectivity in Analytical Chemistry Technical Draft. 2001.

[25] FDA, Bioanalytical Method Validation. Guidance for Industry. 2001.

[26] D.A. Armbruster, M.D. Tillma, L.M. Hubbs, Limit of Detection (LOD)/Limit of Quantification (LOQ): Comparison of the empirical and statistical methods exemplified with GC-MS assays of abused drugs. Clin. Chem. 40 (1994) 1233-1238. 
[27] A. Shrivastav, V.P. Gupta, Methods for the determination of limit of detection and limit of quantification of the analytical methods. Chron. Young Sci. 2 (2011) 21-25.

[28] Morales, M.T., \& Przybylski, R. (2013). Olive Oil Oxidation, in: R. Aparicio, J. Harwood (Eds.). Handbook of Olive Oil. Analysis and Properties, second ed., Springer, New York, 2013, pp. 479-522.

[29] S. Langstaff, R. Aparicio, Defects Wheel for Olive Oil. Applied Sensory LLC. Fairfield, CA. http://www.appliedsensory.com/

[30] IOC, The International Olive Council, Trade Standard applying to Olive Oil and OlivePomace Oil, COI/T.15/NC No 3/Rev. 11. Madrid, Spain, 2016. 
Table 1. Comparison between the results of repeatability and intermediate precision of the methods based on gas chromatography coupled to mass spectrometry (GC-MS) and flame ionization (GC-FID) detectors.

\begin{tabular}{|c|c|c|c|c|c|c|c|c|c|}
\hline \multirow[t]{3}{*}{$\begin{array}{r}\text { Code } \\
\left(R_{t}{ }^{1}\right)\end{array}$} & \multirow[t]{3}{*}{$\begin{array}{l}\text { Volatile } \\
\text { compound }\end{array}$} & \multicolumn{4}{|c|}{$\begin{array}{l}\text { Repeatability } \\
\text { (RSD\%) }^{2}\end{array}$} & \multicolumn{4}{|c|}{$\begin{array}{l}\text { Intermediate precision } \\
(\mathrm{RSD} \%)^{2}\end{array}$} \\
\hline & & ${ }^{3}$ EVOO & EVOO & ${ }^{3}$ LVOO & LVOO & ${ }^{3} \mathrm{EVOO}$ & EVOO & ${ }^{3}$ LVOO & $\overline{\text { LVOO }}$ \\
\hline & & GCMS & GC-FID & GC-MS & GC-FID & GC-MS & GC-FID & GC-MS & GC-FID \\
\hline $1(4.28)$ & Octane & 9.38 & 10.01 & 2.05 & 11.79 & 9.97 & 10.14 & 1.86 & 9.94 \\
\hline $2(5.69)$ & Ethyl acetate & 3.41 & 4.94 & 5.07 & 5.68 & 7.80 & 5.44 & 3.31 & 5.47 \\
\hline $3(6.98)$ & Ethanol & 8.57 & 4.38 & 7.45 & 10.48 & 10.16 & 5.08 & 8.43 & 9.62 \\
\hline $4(8.38)$ & 3-Pentanone & 7.92 & 5.17 & 3.64 & 3.91 & 8.69 & 6.03 & 3.74 & 3.14 \\
\hline $5(8.46)$ & Pentanal & 6.22 & 5.40 & 2.60 & 6.79 & 6.47 & 6.21 & 2.58 & 6.48 \\
\hline $6(10.70)$ & 1-Penten-3-one & nd & 4.19 & 9.29 & 12.73 & nd & 7.87 & 13.28 & 12.69 \\
\hline $7(11.42)$ & 2-Butanol & 8.43 & 16.02 & nd & nd & 10.43 & 17.52 & nd & nd \\
\hline $8(13.93)$ & Hexanal & 9.16 & 4.77 & 4.42 & 5.71 & 9.78 & 5.87 & 6.76 & 6.65 \\
\hline $9(17.87)$ & 1-Butanol & 20.75 & 6.33 & nd & 4.9 & 32.15 & 13.91 & nd & 4.44 \\
\hline 10(19.88) & 2-Heptanone & nd & 9.82 & 1.85 & 6.24 & nd & 11.63 & 1.60 & 6.56 \\
\hline $11(20.08)$ & Heptanal & 6.07 & 5.76 & 2.26 & 4.82 & 6.55 & 6.11 & 2.60 & 5.80 \\
\hline $12(21.37)$ & 3-Methyl-1-butanol & 5.29 & 5.44 & nd & 3.16 & 32.80 & 9.42 & nd & 3.29 \\
\hline $13(21.79)$ & E-2-Hexenal & 4.50 & 7.84 & 3.56 & 5.30 & 6.30 & 8.21 & 1.64 & 5.67 \\
\hline $14(23.35)$ & 3-Octanone & 12.81 & 2.12 & 4.58 & 4.44 & 14.55 & 2.71 & 6.68 & 4.46 \\
\hline $15(25.34)$ & Octanal & 14.24 & 5.85 & 1.65 & 9.03 & 7.46 & 7.48 & 2.93 & 13.94 \\
\hline $16(27.21)$ & E-2-Heptenal & nd & 6.54 & 12.40 & 13.47 & nd & 9.39 & 15.43 & 14.67 \\
\hline $17(27.71)$ & 2-Heptanol & nd & 10.61 & 8.54 & nd & nd & 11.61 & 10.12 & nd \\
\hline $18(28.09)$ & 6-Methyl-5-hepten-2-one & 16.15 & 7.64 & 1.22 & 11.50 & 21.36 & 8.74 & 2.46 & 15.33 \\
\hline $19(28.73)$ & 1-Hexanol & 9.11 & 2.38 & 3.04 & 4.62 & 10.27 & 2.61 & 10.50 & 12.91 \\
\hline $20(30.15)$ & E-3-Hexen-1-ol & 11.85 & 6.57 & nd & 10.35 & 43.50 & 7.03 & nd & 12.63 \\
\hline $21(30.87)$ & Nonanal & nd & 8.01 & 4.02 & 8.98 & nd & 15.86 & 6.97 & 13.75 \\
\hline $22(33.31)$ & 1-Octen-3-ol & 6.13 & 5.73 & 4.35 & 6.57 & 6.52 & 6.42 & 8.00 & 11.36 \\
\hline $23(33.53)$ & Acetic acid & 17.30 & 2.74 & 3.46 & 2.21 & 22.76 & 2.92 & 5.37 & 2.45 \\
\hline $24(37.27)$ & Propanoic acid & nd & nd & 1.99 & 7.01 & nd & nd & 3.90 & 13.74 \\
\hline $25(40.73)$ & Butanoic acid & nd & 9.82 & 3.20 & 6.03 & nd & 17.31 & 7.72 & 9.20 \\
\hline \multirow[t]{2}{*}{$26(44.82)$} & Pentanoic acid & nd & 6.89 & 2.77 & 8.55 & nd & 13.38 & 5.02 & 9.78 \\
\hline & Mean value & 9.85 & 6.60 & 4.25 & 7.26 & 14.86 & 8.76 & 5.95 & 8.92 \\
\hline
\end{tabular}

Note: ${ }^{1}$, Retention time for GC-FID ( $\left.\mathrm{min}\right){ }^{2}{ }^{2}$, Relative Standard Deviation (RSD\%); nd, not detected. ${ }^{3}$, data obtained from reference 8 . 
Table 2. Analytical quality parameters (linearity, working range, limits of detection and quantification, selectivity and sensitivity) of the methods

based on gas chromatography (GC) coupled to mass spectrometry (MS) and flame ionization (FID) detectors.

\begin{tabular}{|c|c|c|c|c|c|c|c|c|c|c|c|c|c|c|c|c|c|c|}
\hline \multirow[b]{2}{*}{ Compound } & \multicolumn{4}{|c|}{ Linearity } & \multicolumn{2}{|c|}{$\begin{array}{l}\text { Limit of } \\
\text { Detection }\end{array}$} & \multicolumn{2}{|c|}{$\begin{array}{c}\text { Limit of } \\
\text { Quantification }\end{array}$} & \multicolumn{4}{|c|}{ Working range $(\mathrm{mg} / \mathrm{kg})$} & \multicolumn{2}{|c|}{$\begin{array}{l}\text { Selectivity } \\
\text { (GC-MS) }\end{array}$} & \multicolumn{2}{|c|}{$\begin{array}{l}\text { Selectivity } \\
\text { (CG-FID) }\end{array}$} & \multicolumn{2}{|c|}{$\begin{array}{l}\text { Sensitivity } \\
\text { (mg/kg) }\end{array}$} \\
\hline & $\begin{array}{c}\mathrm{R}_{\mathrm{aj}}^{21, a} \\
(\mathrm{GC}-\mathrm{MS})\end{array}$ & $\begin{array}{c}\mathrm{R}_{\mathrm{aj}}^{2} \\
(\mathrm{GC}-\mathrm{FID})\end{array}$ & $\begin{array}{l}\mathrm{RSD}_{\mathrm{b}}{ }^{2, \mathrm{a}} \\
(\mathrm{GC}-\mathrm{MS})\end{array}$ & $\begin{array}{c}\mathrm{RSD}_{\mathrm{b}}{ }^{2} \\
\text { (GC-FID) }\end{array}$ & $\begin{array}{l}\text { LOD }^{3, a} \\
\text { (GC-MS) }\end{array}$ & $\mid \begin{array}{c}\text { LOD }^{3} \\
\text { (GC-FID) }\end{array}$ & $\begin{array}{c}\mathrm{LOQ}^{3, \mathrm{a}} \\
(\mathrm{GC}-\mathrm{MS})\end{array}$ & $\mid \begin{array}{c}\mathrm{LOQ}^{3} \\
(\mathrm{GC}-\mathrm{FID})\end{array}$ & $\begin{array}{l}\text { Range }^{a} \\
\text { (GC-MS) }\end{array}$ & $\begin{array}{c}\text { Range } \\
\text { (GC-FID) }\end{array}$ & $\begin{array}{c}\text { Ratio } \\
\text { (GC-MS) }\end{array}$ & $\begin{array}{c}\text { Ratio } \\
\text { (GC-FID) }\end{array}$ & $\begin{array}{c}\mathrm{Rpp}^{4} \\
\text { EVOO }\end{array}$ & $\begin{array}{r}\text { Rnp }^{5} \\
\text { EVOO }\end{array}$ & $\begin{array}{c}\mathrm{Rpp}^{4} \\
\mathrm{EVOO}\end{array}$ & $\begin{array}{l}\text { Rnp } \\
\text { EVOO }\end{array}$ & $\begin{array}{c}(\text { GC-MS) } \\
\times 10^{7}\end{array}$ & $\begin{array}{c}\text { (GC-FID) } \\
\times 10^{4}\end{array}$ \\
\hline Ethyl acetate & 0.987 & 0.999 & 11.84 & 1.56 & 1.18 & 0.15 & 3.92 & 0.51 & $3.92-6.00$ & $0.51-3.00$ & 1.53 & 5.88 & 5.27 & 3.09 & 3.55 & 3.20 & 2.72 & 2.45 \\
\hline Ethanol & 0.998 & 0.995 & 3.50 & $2.24^{b}$ & 0.57 & 1.01 & 1.15 & 3.37 & $1.15-3.00$ & $3.37-15.00$ & 2.61 & 4.45 & 2.92 & 2.26 & 3.14 & 13.90 & 0.30 & 0.69 \\
\hline 3-Pentanone & 0.983 & 0.990 & 4.79 & 6.70 & 0.48 & 1.17 & 1.59 & 3.92 & $1.59-3.00$ & $3.92-6.00$ & 1.89 & 1.53 & 4.26 & 1.50 & 10.28 & 0.55 & 3.35 & 3.55 \\
\hline Pentanal & 0.996 & 0.999 & 1.77 & 2.03 & 0.17 & 0.33 & 0.59 & 1.09 & $0.59-3.00$ & $1.09-6.00$ & 5.08 & 5.50 & 1.50 & 10.17 & 0.55 & 0.76 & 0.29 & 1.56 \\
\hline 2-Butanol & 0.991 & 0.999 & 4.72 & 2.57 & 0.10 & 0.05 & 0.34 & 0.18 & $0.34-1.00$ & $0.18-1.00$ & 2.94 & 5.56 & 5.45 & 6.78 & 1.90 & 2.45 & 0.94 & 2.57 \\
\hline Hexanal & 0.998 & 0.996 & 2.26 & 3.43 & 0.22 & 0.89 & 0.74 & 2.97 & $0.74-3.00$ & $2.97-10.00$ & 4.05 & 3.37 & 4.30 & 2.14 & 3.86 & 0.68 & 1.67 & 1.16 \\
\hline 1-Butanol & 0.972 & 0.999 & $3.03^{b}$ & $1.77^{\mathrm{b}}$ & 0.30 & 0.80 & 0.99 & 2.63 & $0.99-15.00$ & $2.63-15.00$ & 15.15 & 5.70 & 7.76 & 5.05 & 6.16 & 3.32 & 0.96 & 2.65 \\
\hline 2-Heptanone & 0.998 & 0.994 & 2.31 & 3.23 & 0.22 & 0.51 & 0.76 & 1.73 & $0.76-3.00$ & $1.73-6.00$ & 3.95 & 3.47 & 3.17 & 3.04 & 0.39 & 0.69 & 2.27 & 0.23 \\
\hline Heptanal & 0.996 & 0.997 & 0.81 & 0.70 & 0.18 & 0.32 & 0.60 & 1.05 & $0.26-15.00$ & $1.05-15.00$ & 57.69 & 14.29 & 3.04 & 2.58 & 0.69 & 21.28 & 0.75 & 0.62 \\
\hline 3-Methyl-1-butanol & 0.997 & 0.992 & 1.88 & 4.17 & 0.19 & 1.88 & 0.63 & 6.28 & $0.63-3.00$ & $6.28-15.00$ & 4.76 & 2.39 & 8.64 & 1.50 & 6.87 & 2.00 & 0.78 & 1.17 \\
\hline E-2-Hexenal & 0.995 & 0.996 & 1.56 & 3.39 & 0.15 & 0.54 & 0.51 & 1.81 & $0.51-3.00$ & $1.81-6.00$ & 5.88 & 3.31 & 1.50 & 2.85 & 2.00 & 20.29 & 0.62 & 0.48 \\
\hline 3-Octanone & 0.995 & 0.999 & 3.57 & $1.16^{\mathrm{b}}$ & 0.35 & 0.52 & 1.17 & 1.75 & $1.17-3.00$ & $1.75-15.00$ & 2.56 & 8.57 & 2.25 & 1.72 & 8.03 & 3.56 & 0.92 & 0.27 \\
\hline E-2-Heptenal & 0.997 & 0.999 & 4.93 & 1.75 & 1.16 & 0.79 & 3.88 & 2.63 & 3.88-10.00 & $2.63-6.00$ & 2.58 & 3.31 & nd & nd & 1.19 & 1.31 & 0.05 & 0.29 \\
\hline 2-Heptanol & 0.997 & 0.999 & 3.23 & $0.73^{b}$ & 0.32 & 0.33 & 1.06 & 1.11 & $1.06-3.00$ & $1.11-15.00$ & 2.83 & 13.51 & nd & nd & 1.21 & 2.04 & 2.95 & 0.07 \\
\hline 6-Methyl-5-hepten-2- & 0.995 & 0.999 & $4.38^{b}$ & 1.18 & 0.43 & 0.51 & 1.45 & 1.71 & $1.45-15.00$ & $1.71-15.00$ & 10.34 & 8.77 & 2.05 & 4.71 & 9.48 & 12.43 & 0.79 & 0.04 \\
\hline 1-Hexanol & 0.998 & 0.999 & 1.48 & 1.65 & 0.15 & 0.51 & 0.50 & 1.70 & $0.50-3.00$ & $1.70-10.00$ & 6.00 & 5.88 & 1.93 & 3.13 & 12.33 & 9.15 & 1.21 & 0.93 \\
\hline E-3-Hexen-1-ol & 0.985 & 0.990 & 5.60 & 5.21 & 0.86 & 0.82 & 2.86 & 2.79 & $2.86-6.00$ & $2.79-6.00$ & 2.10 & 2.15 & 1.92 & 5.45 & 0.55 & 17.14 & 0.18 & 0.87 \\
\hline Nonanal & 0.987 & 0.991 & 6.35 & 1.89 & 1.14 & 2.41 & 3.98 & 8.05 & $3.98-6.00$ & $8.05-15.00$ & 1.51 & 1.86 & nd & nd & 4.25 & 0.61 & 0.04 & 0.26 \\
\hline 1-Octen-3-ol & 0.998 & 0.998 & 1.17 & 0.76 & 0.32 & 0.74 & 1.07 & 2.46 & $1.07-3.00$ & $2.46-10.00$ & 2.80 & 3.95 & 17.96 & 3.56 & 22.01 & 13.96 & 0.88 & 0.48 \\
\hline Acetic acid & 0.997 & 0.997 & 5.23 & 0.08 & 0.52 & 0.56 & 1.72 & 1.77 & $1.72-3.00$ & $1.77-6.00$ & 1.74 & 3.39 & 4.35 & 5.28 & 8.57 & 3.94 & 0.46 & 0.08 \\
\hline Propanoic acid & 0.998 & 0.990 & 1.75 & $0.54^{b}$ & 0.48 & 1.85 & 1.61 & 6.19 & $1.61-3.00$ & $6.19-15.00$ & 1.86 & 2.42 & nd & nd & nd & nd & 0.56 & 0.54 \\
\hline Butanoic acid & 0.998 & 0.999 & 5.32 & 0.50 & 0.53 & 0.30 & 1.75 & 1.01 & $1.75-3.00$ & $1.01-6.00$ & 1.71 & 5.94 & nd & nd & 2.24 & 9.27 & 0.14 & 0.50 \\
\hline Pentanoic acid & 0.965 & 0.999 & 12.53 & $0.77^{b}$ & 2.54 & 2,34 & 8.49 & 7.79 & $8.49-10.00$ & $7.79-15.00$ & 1.18 & 13.39 & nd & nd & 6.65 & 6.07 & 0.15 & 0.24 \\
\hline
\end{tabular}

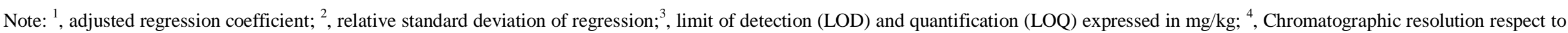

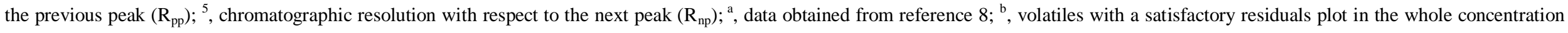

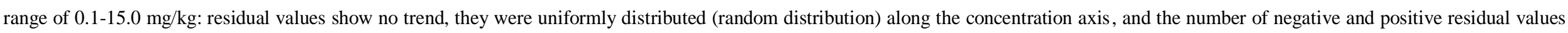
were approximately equal; nd, not detected. 
Table 3. Limit of blank (LOB) of each volatile, range of the lowest concentrations of each volatile producing a signal over its corresponding LOB, limit of detection (LOD) of each volatile with respect to its LOB, odour threshold (OT) of each volatile and its sensory aroma qualifier. Concentration values (mean \pm STD) of twenty-two samples clustered as Lampante Virgin Olive Oils (LVOO), and Extra-Virgin and Virgin Olive Oils (EVOO+VOO).

\begin{tabular}{|c|c|c|c|c|c|c|c|}
\hline Compound & $\begin{array}{l}\text { LOB } \\
\text { (area) }\end{array}$ & $\begin{array}{c}\text { Range }^{\mathrm{a}} \\
\text { (Min-Max) }\end{array}$ & $\begin{array}{c}\text { LOD } \\
(\mathrm{mg} / \mathrm{kg})\end{array}$ & $\begin{array}{c}\mathrm{OT}^{\mathrm{b}} \\
(\mathrm{mg} / \mathrm{kg})\end{array}$ & $\begin{array}{c}\text { Sensory } \\
\text { descriptors }\end{array}$ & $\begin{array}{l}\text { LVOO } \\
\text { (mg/kg) }\end{array}$ & $\begin{array}{c}\text { EVOO+VOO } \\
(\mathrm{mg} / \mathrm{kg})\end{array}$ \\
\hline Octane & 4336 & $0.01-0.05$ & 0.035 & 0.94 & Solvent & $0.51 \pm 0.07$ & $0.17 \pm 0.06$ \\
\hline Ethyl acetate & 1816 & $0.02-0.10$ & 0.061 & 0.94 & Aromatic, ethereal & $1.24 \pm 0.15$ & $0.48 \pm 0.29$ \\
\hline Ethanol & 1645 & $0.01-0.05$ & 0.031 & 30 & Apple, sweet & $8.01 \pm 1.28$ & $3.78 \pm 1.75$ \\
\hline 3-Pentanone & 1000 & $0.01-0.05$ & 0.031 & 70 & Fruity, sweet & $0.04 \pm 0.01$ & $0.47 \pm 0.18$ \\
\hline Pentanal & 1000 & $0.01-0.05$ & 0.020 & 0.24 & Almond, malt & $0.09 \pm 0.01$ & $0.07 \pm 0.05$ \\
\hline 1-Penten-3-one & 6659 & $0.01-0.05$ & 0.034 & $0.7 \times 10^{-3}$ & Pungent, mustard & $0.10 \pm 0.05$ & $0.08 \pm 0.02$ \\
\hline 2-Butanol & 4912 & $0.02-0.10$ & 0.082 & 0.15 & Fruity, medicine & $0.78 \pm 0.34$ & $3.09 \pm 1.12$ \\
\hline Hexanal & 1500 & $0.01-0.05$ & 0.024 & 0.08 & Green, apple, grass & $0.31 \pm 0.03$ & $0.78 \pm 0.09$ \\
\hline 1-Butanol & 2100 & $0.01-0.05$ & 0.029 & 0.40 & Winey & $0.03 \pm 0.01$ & $0.01 \pm 0.01$ \\
\hline 2-Heptanone & 1000 & $0.01-0.05$ & 0.023 & 0.30 & Cinnamon, sweet & $0.02 \pm 0.01$ & $0.00 \pm 0.00$ \\
\hline Heptanal & 10762 & $0.02-0.10$ & 0.062 & 0.50 & Citrus, fatty, rancid & $0.05 \pm 0.01$ & $0.05 \pm 0.01$ \\
\hline 3-Methyl-1-butanol & 12459 & $0.02-0.10$ & 0.079 & 0.10 & Whisky, malt, burnt & $0.96 \pm 0.22$ & $0.44 \pm 0.16$ \\
\hline E-2-Hexenal & 1000 & $0.01-0.05$ & 0.044 & 0.42 & Bitter almond, green & $0.95 \pm 0.25$ & $5.35 \pm 3.78$ \\
\hline 3-Octanone & 1000 & $0.01-0.05$ & 0.030 & 0.75 & Nut & $0.39 \pm 0.09$ & $0.37 \pm 0.22$ \\
\hline Octanal & 1000 & $0.02-0.10$ & 0.083 & 0.32 & Lemon, green, fatty & $0.42 \pm 0.05$ & $0.14 \pm 0.01$ \\
\hline E-2-Heptenal & 1000 & $0.01-0.05$ & 0.062 & $8.3 \times 10^{-3}$ & Soap, fat, almond & $1.37 \pm 0.33$ & $0.62 \pm 0.32$ \\
\hline 2-Heptanol & 1000 & $0.01-0.05$ & 0.025 & 0.01 & Mushroom, chemical & $0.00 \pm 0.00^{c}$ & $0.00 \pm 0.00^{c}$ \\
\hline 6-Methyl-5-hepten-2-one & 7272 & $0.05-0.50$ & 0.145 & 1.00 & Green-fruity, grass & $0.31 \pm 0.04$ & $0.25 \pm 0.20$ \\
\hline 1-Hexanol & 8068 & $0.02-0.10$ & 0.094 & 0.40 & Fruity, alcohol & $0.54 \pm 0.06$ & $2.31 \pm 0.57$ \\
\hline E-3-Hexen-1-ol & 1000 & $0.01-0.05$ & 0.023 & 1.00 & Astringent, bitter & $0.29 \pm 0.05$ & $1.46 \pm 0.33$ \\
\hline Nonanal & 1000 & $0.10-1.00$ & 0.418 & 0.15 & Fat, citrus, green & $8.85 \pm 1.37$ & $3.77 \pm 1.21$ \\
\hline 1-Octen-3-ol & 9143 & $0.10-1.00$ & 0.652 & $1.0 \times 10^{-3}$ & Mushroom, mouldy & $0.02 \pm 0.01$ & $0.00 \pm 0.00$ \\
\hline Acetic acid & 6579 & $0.01-0.05$ & 0.043 & 0.50 & Sour & $7.62 \pm 1.56$ & $2.02 \pm 0.85$ \\
\hline Propanoic acid & 1965 & $0.01-0.05$ & 0.028 & 0.72 & Rancid, soya, pungent & $0.09 \pm 0.03$ & $0.04 \pm 0.02$ \\
\hline Butanoic acid & 17822 & $0.05-0.50$ & 0.313 & 0.14 & Rancid, soya, pungent & $0.96 \pm 0.48$ & $0.08 \pm 0.04$ \\
\hline Pentanoic acid & 2013 & $0.01-0.05$ & 0.040 & 0.60 & Sweat & $0.05 \pm 0.03$ & $0.02 \pm 0.01$ \\
\hline
\end{tabular}

Note: ${ }^{\mathrm{a}}$, in $\mathrm{mg} / \mathrm{kg} ;{ }^{\mathrm{b}}$, data obtained from reference $8 ;{ }^{\mathrm{c}}$, concentration was in the order of magnitude of $\mu \mathrm{g} / \mathrm{kg}$. 


\section{FIGURE CAPTIONS}

Figure 1. (A) Calibration curve of 6-methyl-5-hepten-2-one in the concentration range 0.10-15.00 $\mathrm{mg} / \mathrm{kg}$. (B) Calibration curve of ethyl acetate in the concentration range of $0.10-15.00 \mathrm{mg} / \mathrm{kg}$. The corresponding residual plots are shown as inserts.

Figure 2. Principal component analysis of extra virgin olive oils (E), virgin olive oils (V), and lampante virgin olive oil samples characterized by the sensory defects frostbitten olives (F), winey-vinegary (W), musty-humid-earthy $(\mathrm{M})$, hay-wood $(\mathrm{H})$ and fusty-muddy sediment $(\mathrm{Fu})$. 


\section{FIGURE 1}
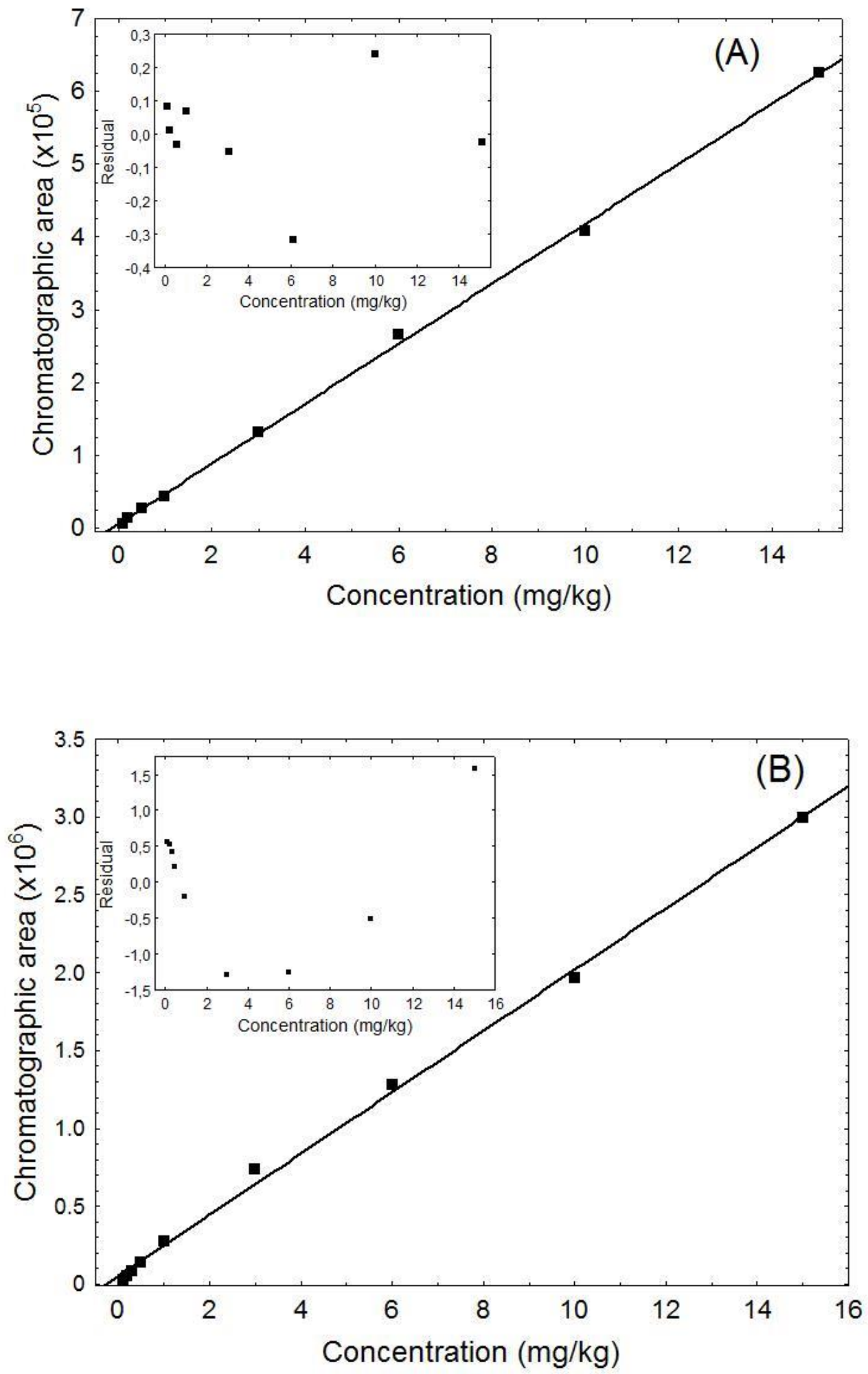


\section{FIGURE 2}

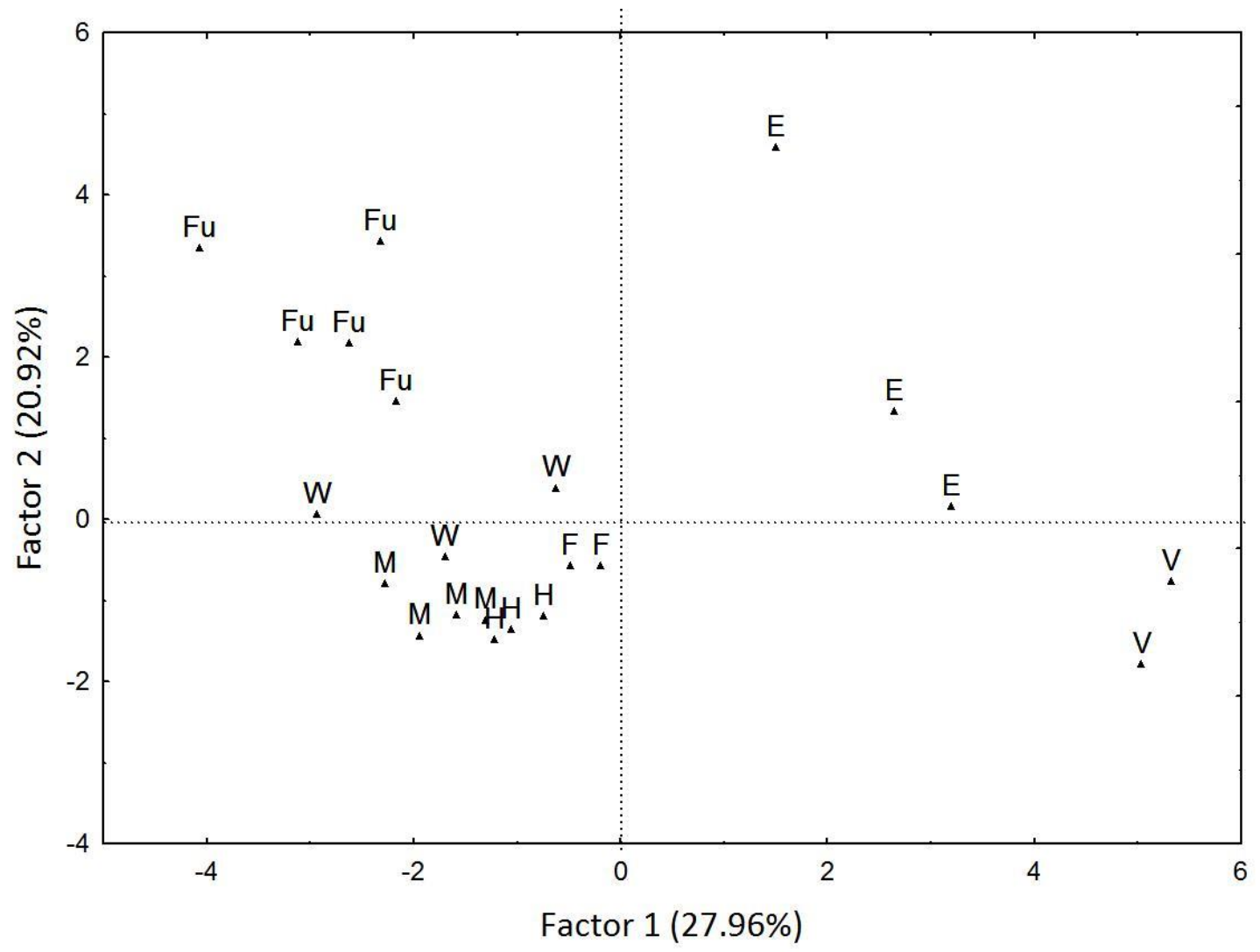

